This item was submitted to Loughborough's Research Repository by the author.

Items in Figshare are protected by copyright, with all rights reserved, unless otherwise indicated.

\title{
Relational-oriented systems engineering and technology tradeoff analysis framework
}

PLEASE CITE THE PUBLISHED VERSION

http://dx.doi.org/10.2514/1.C032079

PUBLISHER

(c) AIAA

VERSION

AM (Accepted Manuscript)

LICENCE

CC BY-NC-ND 4.0

REPOSITORY RECORD

Marvis, Dimitri N., Kelly Griendling, and Charles E. Dickerson. 2019. "Relational-oriented Systems Engineering and Technology Tradeoff Analysis Framework". figshare. https://hdl.handle.net/2134/13083. 
This item was submitted to Loughborough's Institutional Repository (https://dspace.lboro.ac.uk/) by the author and is made available under the following Creative Commons Licence conditions.

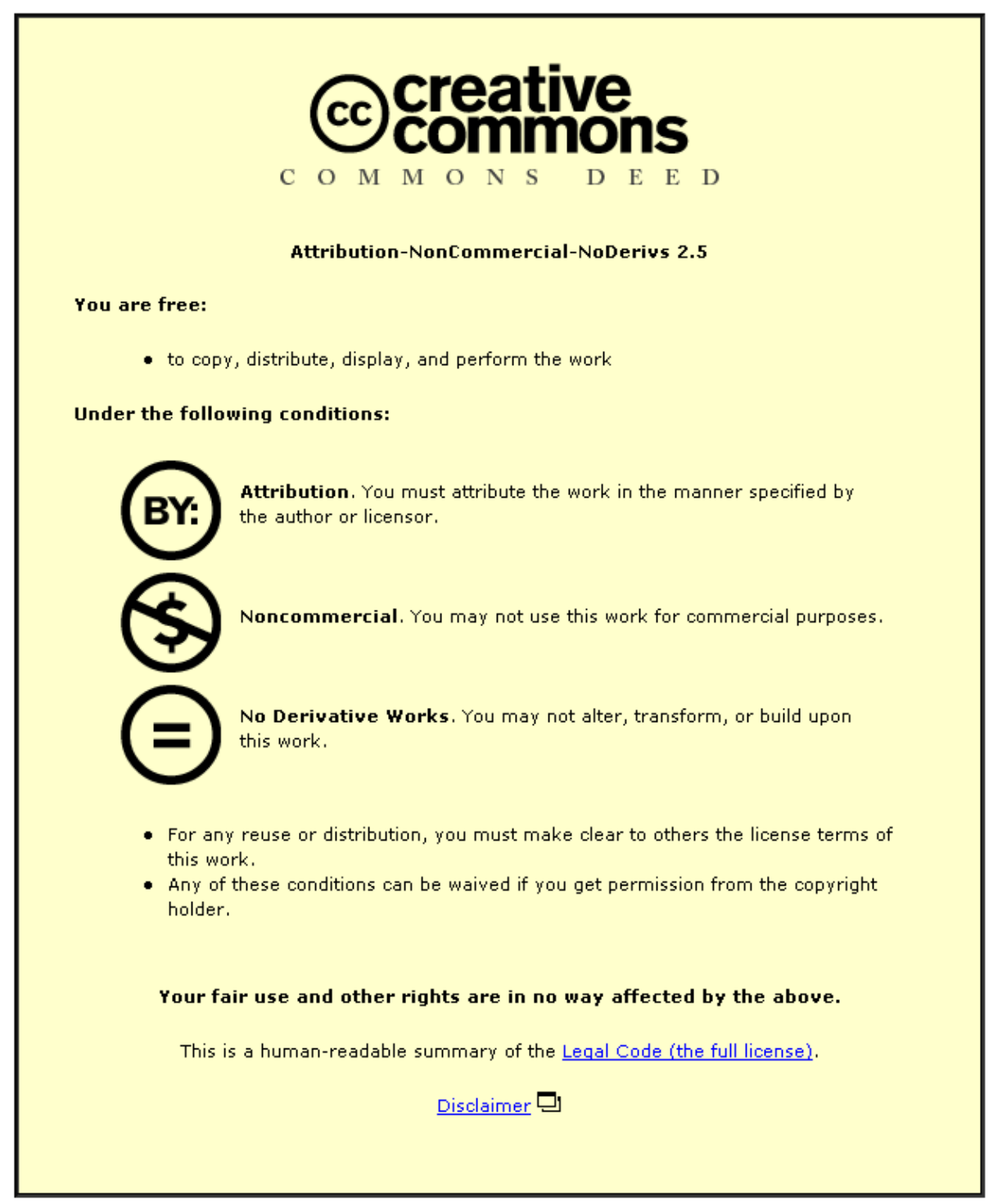

For the full text of this licence, please go to: http://creativecommons.org/licenses/by-nc-nd/2.5/ 


\title{
Relational Oriented Systems Engineering and Technology Tradeoff Analysis (ROSETTA) Framework
}

\author{
Dimitri N. Mavris ${ }^{1}$ and Kelly Griendling ${ }^{2}$ \\ Georgia Institute of Technology, Atlanta, GA 30332 \\ Charles E. Dickerson ${ }^{3}$ \\ University of Loughborough, Leicestershire, LE11 $3 T U$
}

\begin{abstract}
In this paper a new framework for performing early technology tradeoff and design studies, the Relational-Oriented Systems Engineering and Technology Tradeoff Analysis (ROSETTA) framework, is developed and applied to an initial case study to conduct a trade between two candidate technologies for potential application on a commercial jet. ROSETTA leverages the Relational-Oriented Systems Engineering (ROSE) methodology coupled with the exploitation of transformations used in modeling and simulation to create a direct association between the Quality Function Deployment (QFD) methodology and standard quantitative conceptual design space exploration techniques leveraged in technology forecasting and trade studies. This association brings precision to QFD that is model driven and mathematically founded. The approach highlights key deficiencies in QFD when applied to early phase design and technology tradeoff studies for the development of systems. ROSETTA proposes a more rigorous and generalized mathematical framework for conducting generic QFD-type exercises to support decision making in early systems engineering and design, and the advantages of the ROSETTA framework are demonstrated through the application of ROSETTA to a small-scale aerospace technology tradeoff. ROSETTA provides a means to begin to formalize and strengthen the relationship between QFD, modeling and simulation, and theoretical mathematics, and allow translation between these three approaches to engineering problems.
\end{abstract}

\footnotetext{
(Nomenclature entries should have the units identified)

$b \quad=\quad$ constant coefficient in response surface equation

$m \quad=\quad$ metric
}

Nomenclature

\footnotetext{
${ }^{1}$ Boeing Chaired Professor and Director of Aerospace Systems Design Laboratory, School of Aerospace Engineering, and AIAA Fellow.

${ }^{2}$ Research Engineer II, School of Aerospace Engineering, and AIAA Professional Member

${ }^{3}$ Professor, School of Electronic, Electrical, and Systems Engineering
} 


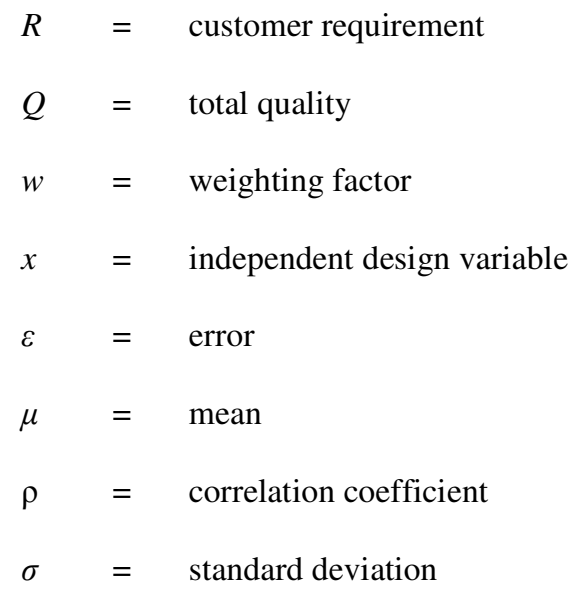

\section{Introduction}

THE Rosetta stone provided a means to translate between the Greek, Hieroglyphics, and Egyptian demotic languages by having the exact same text (a decree) repeated in all three languages. The Rosetta stone was the key to deciphering hieroglyphics by using the other two languages which were well known. In the same way, the Relational-Oriented Systems Engineering and Technology Tradeoff Analysis (ROSETTA) Framework has been developed to provide a means for translation between theoretical mathematics, subject-matter expert driven analysis, and modeling and simulation, by representing a single problem using all three types of analysis and highlighting the commonalities and differences resulting from the different representations of the problem. Using this relational-oriented perspective allows for a critical examination of commonly used early-phase engineering techniques and these observations will be used to suggest an improved and more rigorous framework that leverages the information commonly collected in early phase engineering to increase the accuracy of initial predictions. In particular, this work examines the initial prediction of which engineering characteristics or metrics will have the most impact on meeting the overall requirement set.

The subject matter expert-driven analysis used is in the form of the quality function deployment (QFD). The traditional QFD can be constructed from the combination of the seven Management and Planning Tools and is typically used in the early phases of Systems Engineering to elicit tacit knowledge from subject matter experts and use that knowledge to identify key design drivers and trades [1]. The QFD is made of one or more hierarchical House of Quality (HOQ) tools. The HOQ that is used here maps a set of customer requirements to a set of engineering characteristics and uses the mapping to better understand the overall relationships between the requirements and between the engineering characteristics. The output of a QFD is an estimation predicting which engineering variables will have the greatest impact on the ability to meet requirements. Although QFD was initially developed for studying manufacturing processes, it has been applied to a number of design activities, including being applied in the F-35 technology selection [2]. 
Using modeling and simulation can provide a quantitative and more objective approach to obtaining the same information. In the ROSETTA framework, the behavior of the modeling and simulation environment in the area of interest will be captured by using the response surface method (RSM) [3] to wrap a set of surrogate models, specifically response surface equations (RSEs), around the modeling and simulation environment. Because the RSEs are simply a polynomial multivariate regression of the model, the behavior of the modeling and simulation environment can be explored through the behavior of these simple mathematical functions. These RSEs will enable rapid (near real-time) execution of the simulation, and will allow trends across the design space to be easily understood and quantified, thus helping to determine the strengths of the relationships represented in the body of the HOQ. The RSE approach also allows for a Monte Carlo Simulation (MCS) to be rapidly conducted, and the results can be used to obtain information about the correlations of the response variables that are found in the roof and the greenhouse of the HOQ. The results of this environment can be compared the QFD approach, highlighting key areas that need to be improved when using QFD for engineering design and technology tradeoff analysis. The ROSETTA framework developed in this paper is proposed to fill these identified gaps.

A more formal understanding of the information developed using either the QFD approach or the M\&S approach can be obtained by representing the relationships mathematically. The Relational-Oriented Systems Engineering (ROSE) viewpoint presented by Dickerson and Mavris [4] is used to enable this formalization. Rooted in the first-order model theory of mathematics but adapted to engineering practice, ROSE is a model driven general systems methodology that employs a principle of model specification and relational transformation for the purpose of system specification, design, and analysis. From the relational viewpoint, the specification of a model associated with a system is focused on the relations of the entities of the system, the architecture and the transformations of both. The mathematical foundation for ROSE supports the rigorous development of structures for the design of systems and the assemblage of systems of systems that adhere to the principles of structured analysis and design.

ROSE enables a calculus-based representation of HOQ that applies rigorous transformation formulae to describe the relationships in the HOQ and is used to understand the nature of the HOQ, its implications in the overall QFD approach, and to help determine what information is required in an equivalent quantitative approach. At the same time, this mathematical approach can provide a direct mathematical translation between the information contained within the RSEs and the information elicited by the SMEs. This comparison is used to identify several challenges in the application of QFD to engineering design and technology tradeoff problems. To address these challenges, ROSETTA proposes a mathematical model that leverages the basic structure of the QFD while allowing for more complicated relationships to be captured and included in the initial analysis of the importance of the engineering characteristics. 
In order to explain the application of ROSETTA to a technology tradeoff, the following notation will be used: Customer requirements will be considered as the future goals that a chosen technology portfolio is trying to meet, and will (from this point forward) be referred to as the 'R-space'; The engineering characteristics are the metrics on which the technologies will act, and are called the 'm-space'; and the fundamental design variables of the system that will map to the metrics at the next lowest level will be called the ' $\mathrm{x}$-space'.

Stated succinctly, ROSETTA leverages the ROSE methodology to both begin to formalize and generalize the QFD and make it more applicable to aiding with technology and design tradeoffs. ROSETTA allows for more complicated relations and transforms to be captured and used in the QFD, and allows for the information contained in the roof or greenhouse of the QFD to be included in the determination of the QFD results. In order to develop and describe the ROSETTA framework, four claims will be made and substantiated in this paper:

Claim 1: The use of transformations in existing M\&S methods can be exploited to strengthen QFD. One of the fundamental underpinnings of the ROSE methodology is the use of transformations of models from a relational viewpoint. The HOQ of QFD has a structure that lends itself to integrating standard modeling and simulation practices through the rigorous approach to using transformations provided by ROSE. This will be demonstrated and described more fully throughout this paper.

Claim 2: The qualitative approximation provided by QFD is concerned with the same information that is obtained quantitatively in $M \& S$. Although QFD and M\&S look distinctly different on the surface, these two approaches are attempting to discover similar information about a system to be designed. The contexts in which each are applied and the fidelity of the results are different, but the fundamental goal of each is to gain insight into a system design problem by mapping design variables to design requirements. As such, these approaches provide two different means to collecting similar information.

Claim 3: ROSETTA applies ROSE to QFD to create a more formalized structure where qualitative information can be integrated with quantitative results when available. The formalization of the relationships and transformations using ROSE allows the development of a more generic and flexible framework to describe both qualitative and quantitative representations of a system. This framework can be used for both qualitative and quantitative representations to gain insight into engineering design problems and to preserve analysis results for later use.

Claim 4: A more formal representation of the QFD using ROSE allows improvements in how this existing method can be used to support engineering decision making. The case study presented in this paper shows a simple example of how the application of ROSETTA can improve the decision making process for a simple aircraft technology tradeoff study. This claim is substantiated by the application of ROSETTA to a case study using a practical aerospace technology tradeoff problem, highlighting the benefits of the framework. 


\section{Claims 1 and 2}

\section{A. Qualitative Analysis}

Qualitative analysis often leverages QFD, which is a widely practiced quality engineering technique that has been credited with significant reductions in cost and product development time, as well as increases in productivity when applied correctly in product development [5]. QFD uses information elicited from subject matter experts (SMEs) to map customer needs and requirements to the engineering characteristics that are required to fulfill those needs with the goal of understanding the relationships between the engineering characteristics and between the requirements. It is used to help identify which capabilities and enablers are most important to helping answer top level requirements. It provides a methodical translation between the 'voice of the customer' and the technical requirements of the engineer. QFD has been credited with the quality revolution in microelectronics and automotive sectors [5], [6]. This application of the QFD has been repeated in many product development sectors, including consumer electronics, home appliances, clothing, and many others [6]. The QFD has also been applied to address and study a variety of other topics, such as the link between manufacturing flexibility and market requirements [7] and university course design [8], among many others. However, one important note is that QFD assumes linear relations by the nature of the use of constants to populate the interior of the QFD. Case studies in the literature have not verified that this assumption applies before using QFD, thus calling into question the validity of these applications of QFD and the associated results.

A QFD is constructed by consulting with subject matter experts (SMEs) and customers to create one more HOQ, which is the basic design tool of the QFD approach. The QFD includes the set of planning and communication routines that help develop and use these HOQs. The HOQ is a conceptual map that provides a means for planning and communication between two groups of people with different problems and responsibilities (often customers and engineers) [6]. To create an HOQ, the first step is to determine the customer requirements and the engineering characteristics needed to fulfill them. There are several compartments that comprise an HOQ, as shown in Fig. 1. The requirements are given in compartment 'A', and the engineering characteristics in compartment ' $\mathrm{E}$ '. The customer puts weightings on the requirements (usually on a linear 1-10 scale) in compartment 'B'. For each engineering characteristic, the direction of improvement ('larger is better', 'smaller is better', 'nominal is better') is identified in compartment ' $\mathrm{D}$ '. To fill in the body (compartment ' $\mathrm{C}$ ') of the HOQ, the SMEs create a mapping between the requirements and the engineering characteristics describing how strongly each engineering characteristic is related to each requirement. In other words, the SMEs are answering the question, "In general, how much impact does this engineering characteristic have on the ability to meet this requirement?" The answers are given in the form of 'strong impact', 'medium impact', 'weak impact', or 'no impact'. These answers are then translated to a non-linear numerical scale with 9 corresponding 
to 'strong impact', 3 corresponding to 'medium impact', 1 corresponding to 'weak impact', and 0 corresponding to 'no impact'. This is an example of one scale that is commonly used, but the choice of scale is dependent on the user and the application of the HOQ. It is common, however, for the chosen scale to be non-linear, such that a strong relationship carries significantly more weight than a weak one. For each engineering characteristic (each column of the HOQ), its total importance is calculated by multiplying the weighting of each requirement by the impact score for that engineering characteristic to that requirement, and then summing the product. This is done for each engineering characteristic, and the results are given in compartment 'I' [5].

Since it is recognized that the engineering characteristics may be correlated to one another, and the requirements may be correlated to one another, these correlations are captured in the HOQ as well. The correlations between requirements are given in compartment ' $\mathrm{H}$ ', which is sometimes referred to as the 'greenhouse'. The correlations between engineering characteristics are given in compartment ' $\mathrm{G}$ ', which is sometimes referred to as the 'roof'. The correlations are identified by the subject matter experts, and each identified correlation is determined to be 'strong negative' (corresponding to a score of -3), 'negative' (corresponding to a score of -1), 'positive' (corresponding to a score of 3), or 'strong positive' (corresponding to a score of 9). These correlations can be used to help identify where critical design tradeoffs may be expected to occur. Although scores are associated with the correlations, these scores are never used in any kind of calculation. Alternatives are listed in compartment 'F', and evaluated with respect to how well they meet each of the requirements [5].

It is also important to note that the implementation of QFD often includes the hierarchical linking of several HOQs. To accomplish this, the engineering characteristics from one HOQ become the requirements for the next HOQ, and the requirement weightings are the output scores from the previous HOQ. The greenhouse for the secondary QFD would be the same as the roof of the first QFD. The engineering characteristics used for the second QFD represent the next level down in detail. SMEs can then be again consulted to determine the mappings and aid in the associated voting, although it is often the case that a different set of SMEs will be required. This deployment can be repeated until the desired level of fidelity is achieved in the engineering characteristics [5]. The general QFD process is illustrated in Fig. 2.

Since the QFD relies on a series of mapping matrices relating requirements and engineering variables, engineering variables to themselves, and requirements to themselves, it is a simple matter to see that these can be viewed as transformations. In fact, these can be viewed as relational transformations according to set theory. The greenhouse and the roof of the QFD represent relations on the set of requirements and the set of engineering characteristics respectively, and the body of the QFD is the transformation between the requirements set and the engineering characteristics set. 


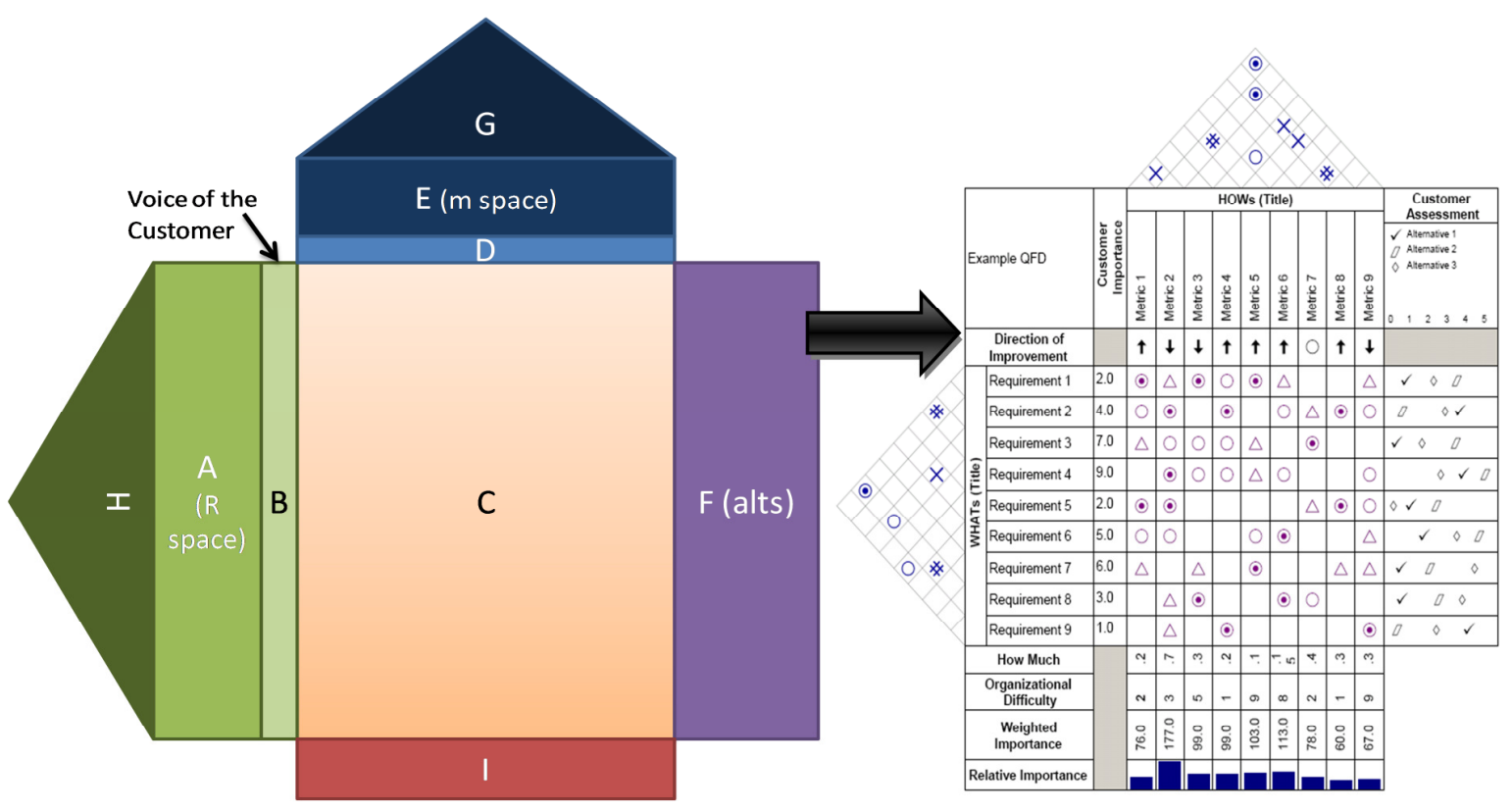

Fig. 1 Generic HOQ structure and example HOQ, showing how the generic graphic used here translates to the common view of a QFD.

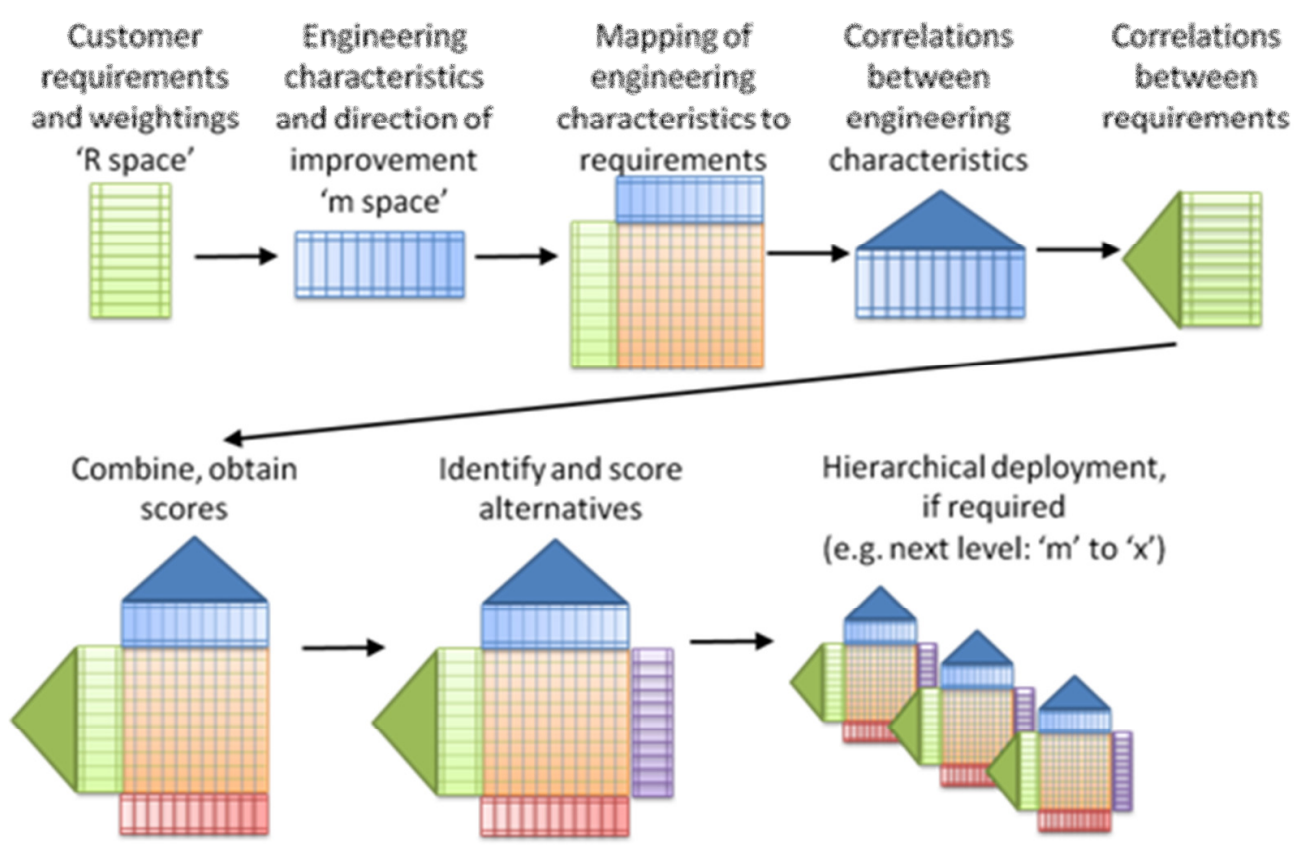

Fig. 2 Generic QFD Process. Note that as the QFD is constructed, mapping matrices similar to those used in ROSE are being constructed and can be used to provide the relations and relational transforms required by ROSE.

\section{B. Quantitative Analysis}

The QFD uses a subjective approach to develop the relations between customer requirements, metrics, and design variables. It is possible (and in fact is common practice in subsequent phases of design) to develop these same relationships through modeling and simulation (M\&S). Developing these results via M\&S requires that the M\&S setup must produce information 
about the strength of the relationship between engineering characteristics and requirements, the correlations between the requirements, and the correlations between the engineering characteristics. To do this, it is first important to realize that for modeling and simulation, the requirements need to be measureable and a function of the engineering characteristics. If this is not the case, it may be necessary to decompose the requirements and engineering characteristics further to obtain measurable requirements. However, for the purpose of this paper, it will be assumed that the requirements are measurable outputs of the M\&S environment. Thus, the engineering characteristics are actually the metrics in the quantitative space. These metrics are in turn a function of the independent design variables, which would be captured qualitatively through QFD deployment to a second level. This simple observation provides important guidance for QFD creation. First, requirements must able to be translated to measureable quantities that are a function of metrics. All of the metrics that influence all of the requirements must be included in the HOQ in order to fully capture the relationships and rank the metrics fairly. The second HOQ deployed in the QFD should map metrics to independent design variables, and again, must include all of the design variables that impact the metrics.

However, it is also important to note that M\&S generally does not use this type of decomposition. In models, the requirements and metrics are both tracked as outputs to the simulation, and the independent design variables are the inputs to the simulation. Thus, to a modeling a simulation environment, there is unlikely to be any difference between a requirements and metric, as both can be tracked as outputs to the simulation. However, many parametric M\&S environments have the ability to include multipliers, also sometimes referred to as "k-factors" as inputs to the model. These multipliers allow the user to adjust the value of intermediate metrics without adjusting the value of design variables. These have been used to model technology infusion. For example, these k-factors could be used to simulate the impact of choosing composite materials over aluminum for an aircraft wing by placing a multiplier on the wing weight that simulates the impact of the new material. A comparison of the decomposition used by a QFD and the decomposition used by M\&S is shown graphically in Fig. 3.

The desired relationships can be obtained by using a modified version of the M\&S approach taken in the Technology Identification, Evaluation, and Selection (TIES) Method. Details about this methodology can be found in Kirby \& Mavris [9]. In order to obtain both the relationships between the requirements and the metrics, as well as between the metrics and the design variables, two parallel M\&S setups are developed, one dedicated to each set of relations (equivalently, each HOQ in the QFD deployment). Producing the needed information requires a very thorough exploration of the full design space (within ranges of interest), so a parametric environment is required to capture the behavior. In order to enable the large numbers of cases required to develop this, surrogate models are created from the actual codes. These surrogates are extremely accurate to the behavior of the original codes, but are a mathematical equation (regression) that runs in a fraction of the time. Additionally, since these surrogates are typically continuous functions through the design space, it is possible to visually and mathematically examine the shape of the design space. When possible, response surface equations (RSEs) are the preferred form of these surrogates because 
they are simple to create and intuitive relative to other forms of surrogates (such as neural networks or Gaussian processes). However, any form of surrogate will work for this approach. The general form of a second-order RSE is shown in Equation (1) [3] where the error term $(\varepsilon)$ is assumed to have a normal $(\mathrm{N}(0,1))$ distribution.

$$
R=b_{o}+\sum_{i=1}^{k} b_{i} x_{i}+\sum_{i=1}^{k} b_{i i} x_{i}^{2}+\sum_{i=1}^{k-1} \sum_{j=i+1}^{k} b_{i j} x_{i} x_{j}+\varepsilon
$$

The surrogates are created by selecting an appropriate design of experiments (DoE) and executing this on the actual codes. This subset of cases is used to perform the regression required to develop the surrogate models. The form of the DoE is chosen based on the expected form of the surrogate models. If the assumed type of surrogate model does not meet the desired accuracy, then a new form is assumed, a new DoE is created, and the process is repeated until acceptable surrogates are created. These surrogates can be used to determine the relationship between independent variables and the responses. This is done using a sensitivity analysis, such as the one shown in Fig. 4, which displays all of the sensitivities between the metrics and the requirements and is created from the surrogates. Each of the boxes contains the sensitivity of one metric (on the bottom) to one response (on the side), and is dependent on the assumed values for the other metrics (indicated by the vertical dashed line and the number shown above the metric names). In the sensitivity analysis, the red crosshairs can be moved, instantly updating the sensitivities and the predicted responses. This highlights an important difference between the results of the M\&S and the predictions made in the corresponding HOQ. While the HOQ estimates are static representations of the strength of the relationships, the results of a sensitivity analysis show that any time there is coupling between metrics, the sensitivity between a metric and requirement can vary as a result of the assumed values of the other metrics. This implies that the sensitivities cannot be accurately represented by a single value, but rather are partial derivatives that are a function of the other metrics. Thus, it is important for the ROSETTA framework to extend the current QFD approach to be able to capture these sensitivities more generally.

A second key difference between the sensitivity analysis shown here and the QFD approach is that the surrogates are only valid within the chosen ranges for the independent variables. In the SME-based QFD approach, ranges and assumptions are not specified and documented within the framework. It is possible that an independent variable and a response have a strong relationship in general, but have little or no relationship within a given range. An example of this can be seen in Fig. 5, which shows the general relationship of the aircraft drag coefficient against the Mach number. Overall, Mach number has a strong relationship with drag coefficient. However, in the subsonic region, the relationship is minimal. The answer given by a subject matter expert regarding the magnitude of this relationship may vary depending on his assumptions about the given problem and how much information has been provided. This is an important consideration when framing the questions that a SME is expected to answer for the QFD exercise. 
QFD Deployment

$\mathrm{m}$

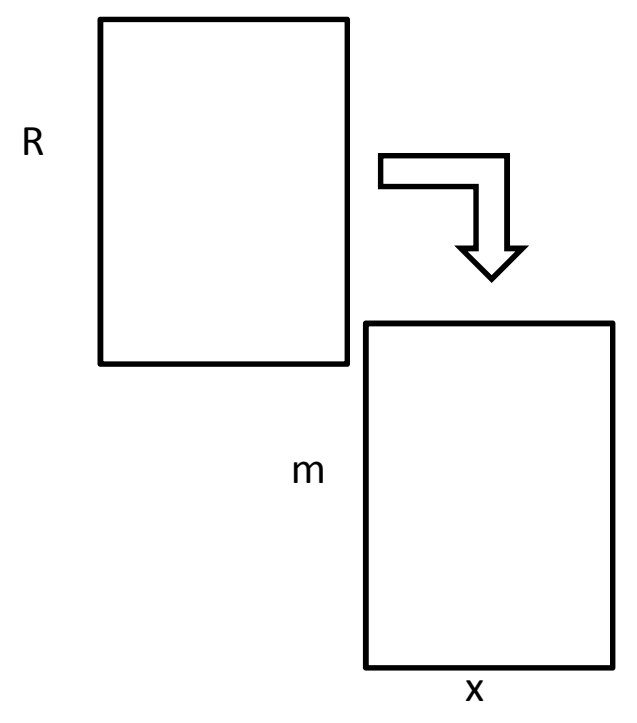

M\&S Association or Transformation

$\mathrm{R}$

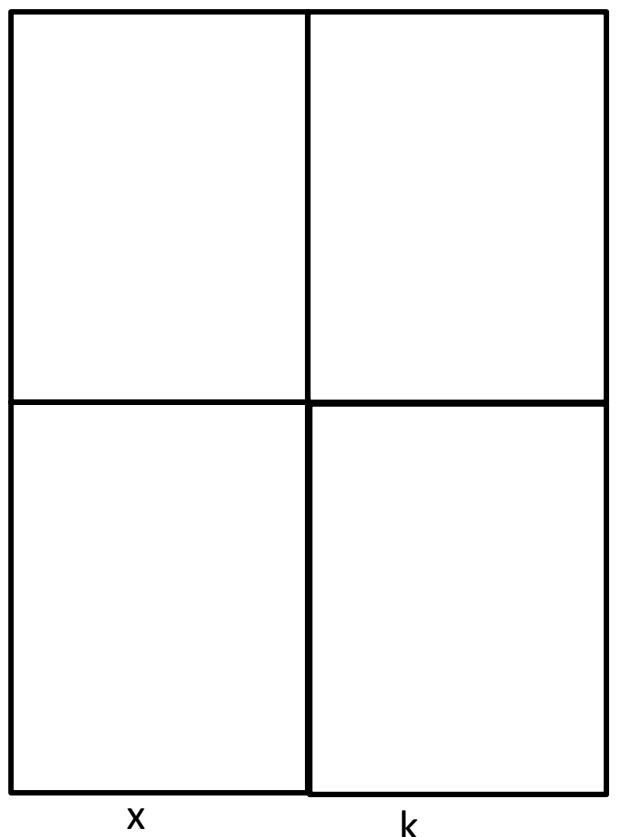

Fig. 3 Comparison of QFD and M\&S Decomposition of a Problem

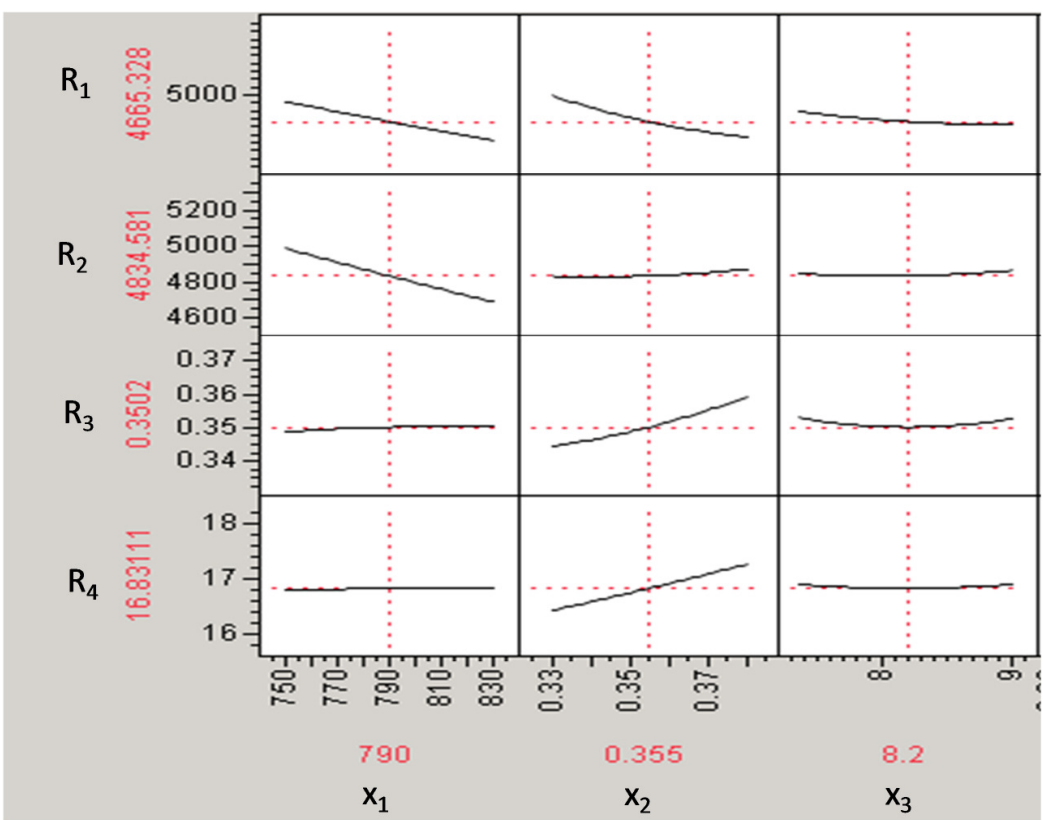

Fig. 4 Sub-section of a notional sensitivity analysis. This shows three metrics on the $x$-axis against four requirements on the $y$-axis. The sensitivity analysis depicts the function that acts as the quantitative transformation between the metrics and requirements. 


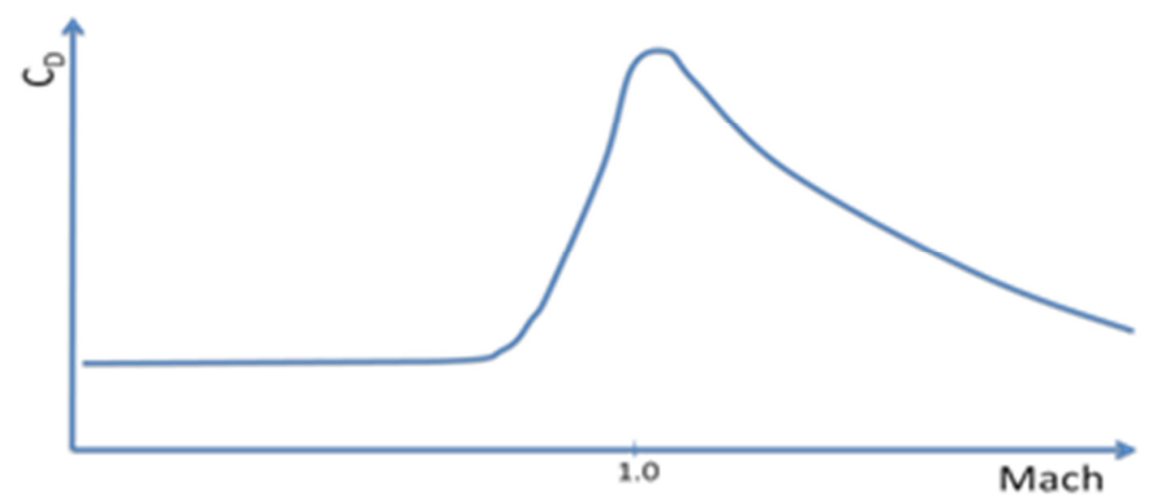

Fig. 5 Notional Plot of aircraft drag vs Mach number

Once these surrogates are developed, a Monte Carlo Simulation (MCS) can be executed by placing random uniform distributions on each of the independent variables and running a large number of cases (often 10,000 or greater). Because this MCS will cover the full design space, it can be used to obtain the correlations between the responses using multivariate analysis.

A visual examination of the results of this MCS through the use of multivariate plot, as shown in Fig. 6, suggests that correlations will not fully describe the relationships between requirements or between metrics. Each box of the multivariate plot shown represents one requirement mapped against another. The full matrix then gives every requirement mapped against every other requirement. The thick blue outline corresponds to the quantitative version of the roof of the QFD.

In each of the boxes, every case of the MCS is represented. The density ellipses encircle $95 \%$ of the cases, assuming a joint normal distribution. The numbers in the upper left-hand corner of each box give the correlation coefficient between those two requirements. Correlation gives a measure of the linear dependence between two variables, and is expressed on a -1 to 1 scale using Pearson's correlation coefficient. The equation for the correlation coefficient is given in Equation (2).

$$
\rho_{X, Y}=\frac{\operatorname{Cov}(X, Y)}{\sigma_{x} \sigma_{Y}}=\frac{E\left[\left(X-\mu_{X}\right)\left(Y-\mu_{Y}\right)\right]}{\sigma_{x} \sigma_{Y}}
$$

A correlation coefficient close to -1 implies a very strong negative correlation. If it is close to 1 , this implies a strong positive correlation, and if it is close to 0 , this implies no correlation. While a magnitude of the correlation coefficient close to 1 implies the existence of a relationship between two variables, this only measures the strength of the linearity of the relationship. The correlation coefficients are shown numerically in Fig. 6, and also represented visually by the blue ellipses. In addition to the correlation, the sensitivity of one parameter to the other is also captured in the multivariate analysis. This is represented by the slope of a line fit through the points. The sensitivities are shown visually in Fig. 6 by the red lines. These observations show 
that is important that the ROSETTA framework be able to more accurately capture the relationships in the greenhouse and the roof of the HOQ, allowing for the sensitivities between parameters to be captured rather than just the correlation.

Determining the sensitivities and correlations between the metrics require that this process be repeated one level lower of detail, where the metrics act as the responses and a new set of independent variables that will influence the engineering characteristics. This is the same information that would be required to create the second HOQ in a hierarchical QFD implementation. When the above process is repeated for this set of variables, the resulting multivariate analysis will provide the correlations and sensitivities between the metrics. The full M\&S approach is summarized in Fig. 7.

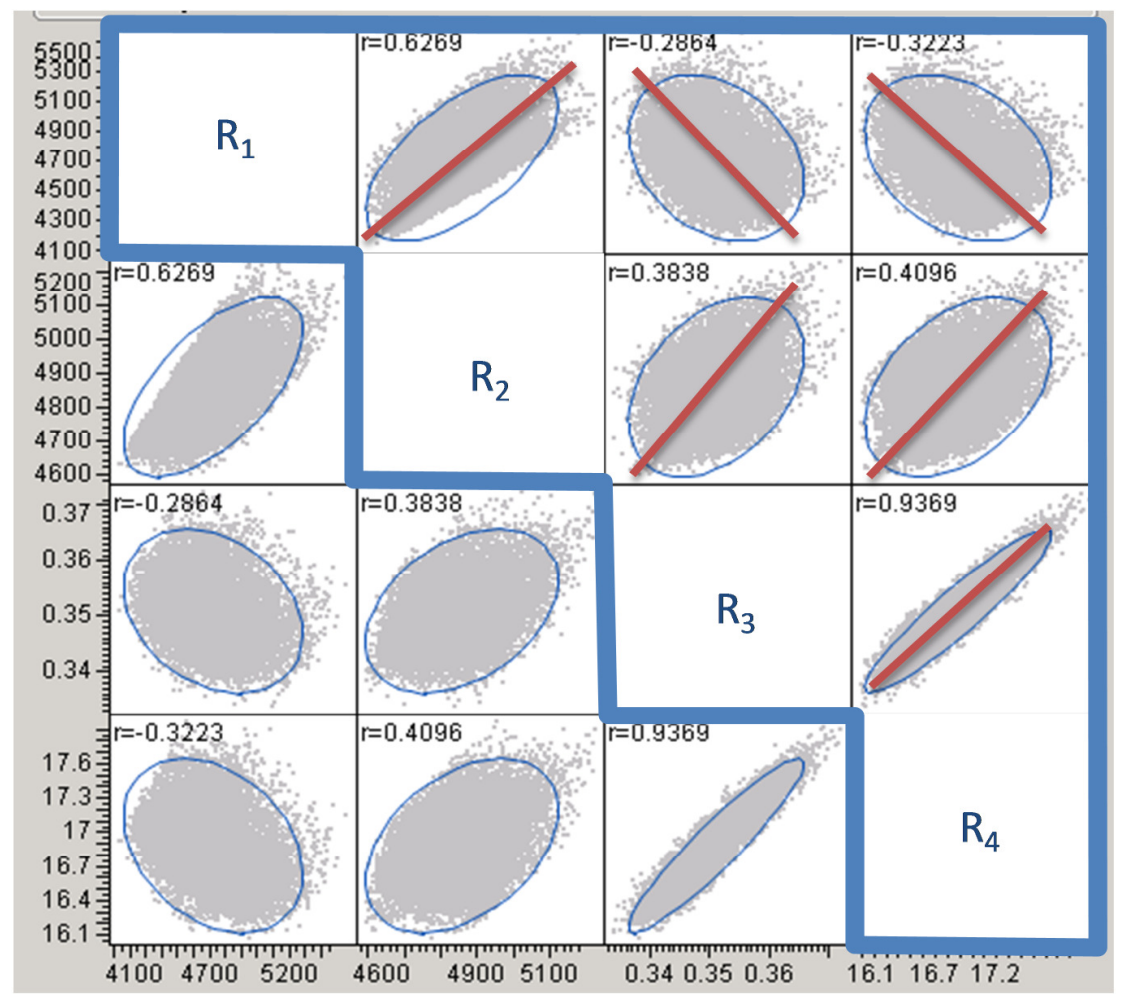

Fig. 6: Example notional multivariate analysis including correlation coefficients and density ellipses 


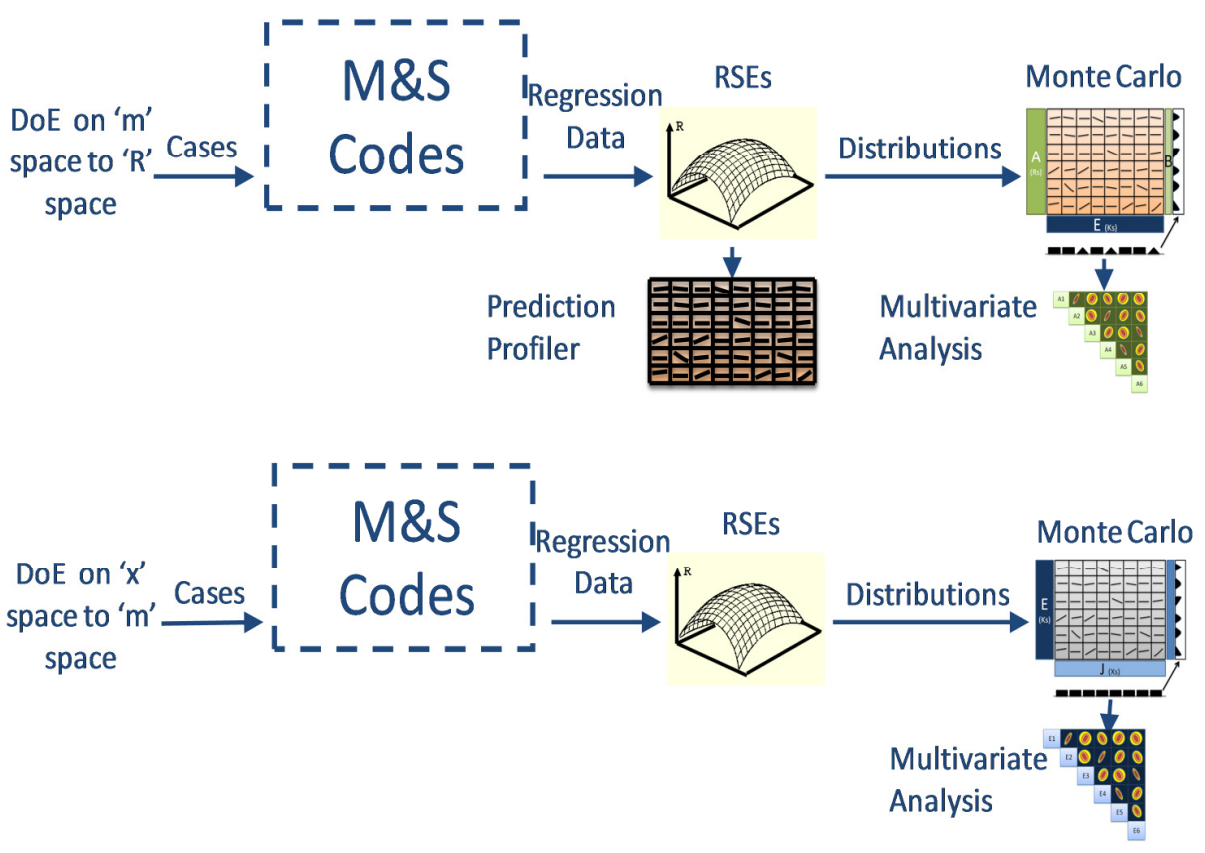

Fig. 7: Modeling and Simulation Analysis Setup.

Furthermore, Multivariate Analysis of Variance (MANOVA) can be used to obtain the impact scores typically seen along the bottom of the HOQ. MANOVA is generalized form of analysis of variance (ANOVA), which allows a user to simultaneously evaluate mean differences on two or more dependant variables. Performing a MANOVA can help uncover which factors are most important to the variability of all responses. MANOVA can be a particularly powerful analysis technique if it is known or suspected that the dependant variables are correlated [10]. This is the quantitative analogy to the weighted scores that provided in compartment 'I' of the HOQ. Recognition of the similarities between the formal MANOVA approach and the more informal HOQ approach to determining factor weightings highlights the potential for use of QFD as a very early, rudimentary screening test. This use of QFD will require well formed questions to be posed to the SMEs with a clear understanding of assumptions and limitations on the design ranges. It will also require an appropriate choice of scale within the body of the QFD to ensure that priorities of factors are not falsely reversed.

The surrogate models developed in this approach are mathematical formulas representing the transformation between engineering variables and requirements, and the multivariate analysis provides the transformations between the requirements and themselves and the engineering variables and themselves. These are the same transformations that are represented in the QFD approach discussed in this research. In fact, Fig. 8 shows the analogy between the modeling and simulation results and the relations that are described in the QFD. 


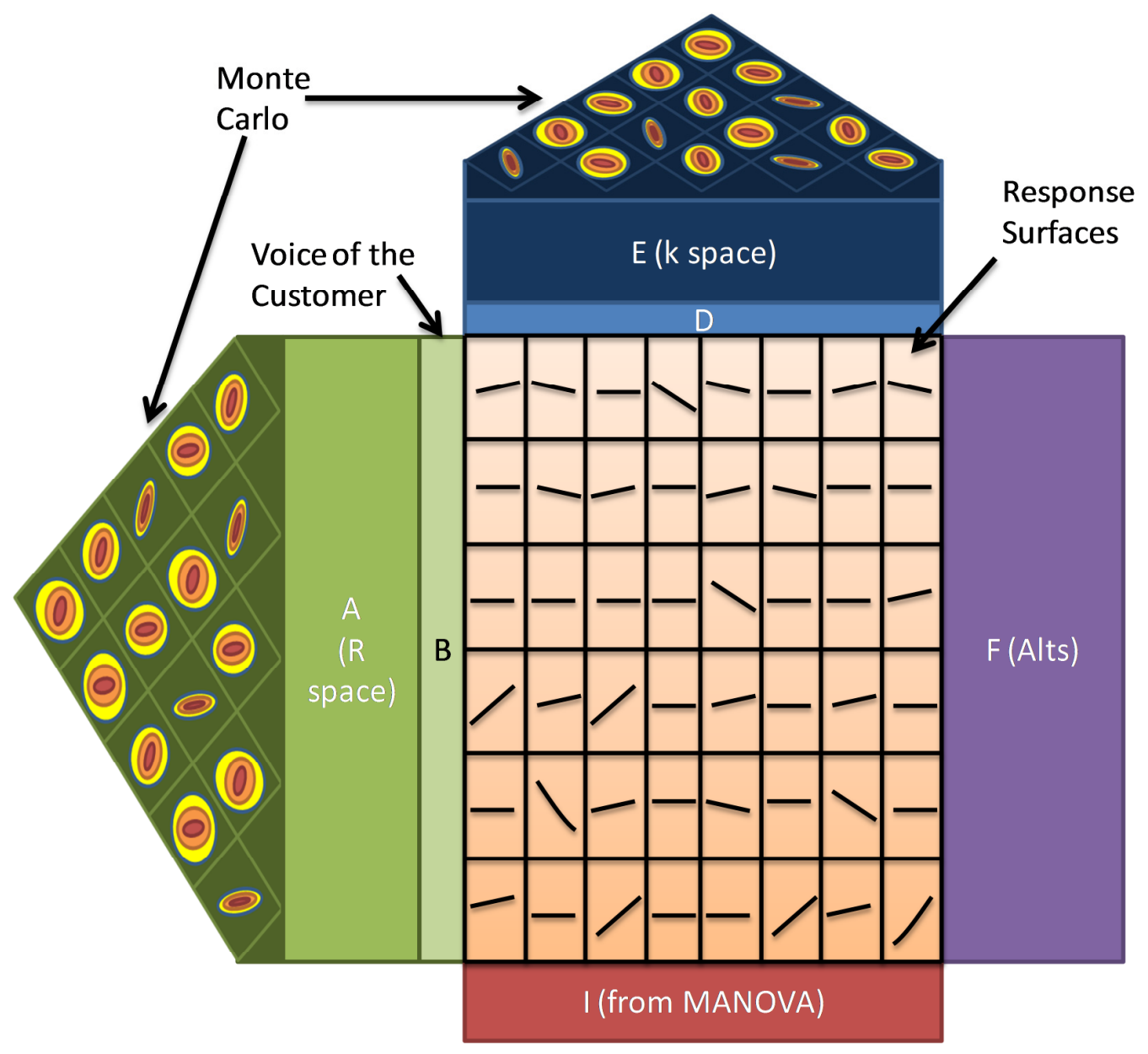

Fig. 8: Analogy between M\&S results and QFD. The sensitivity analysis is shown in the body, and the multivariate analysis comprises the roof and the greenhouse. MANOVA is used to obtain the importance rankings of the metrics to the total requirement space.

\section{Claim 3}

A better understanding of the relationship between the QFD and Modeling and Simulation can be obtained by understanding the mathematical nature of the relationships that the SMEs are attempting to subjectively express in the HOQ. ROSETTA applies ROSE to QFD to create a more formalized structure where qualitative information can be integrated with quantitative results when available. The transformation formulae presented here are an extension on the model transformation rules presented in Dickerson and Mavris [4] [11]. For the purpose of this discussion, the requirements will be referred to as members in the set of dependant variables $\left\{R_{1}, R_{2}, \ldots, R_{n}\right\}$, and the metrics will be referred to as members in the set of independent variables $\left\{m_{1}, m_{2}\right.$, $\left.\ldots, \mathrm{m}_{\mathrm{p}}\right\}$. In the body of the HOQ (the ' $\mathrm{C}$ ' matrix in Fig. 1), the SMEs are commenting on the strength of the relationship between a given $r_{i}$ and a given $m_{k}$. Mathematically, this matrix is the relational transformation between the r-space and the $m-$ space. For the $(\mathrm{i}, \mathrm{k})$ cell in the ' $\mathrm{C}$ ' matrix, this transformation is represented by the slope of the partial derivative, $\delta \mathrm{R}_{\mathrm{i}} / \delta \mathrm{m}_{\mathrm{k}}$. 
However, the assertion that this partial derivative fully and completely represents the sensitivity of $R_{i}$ to $m_{k}$ is dependent on the assumptions that the $\mathrm{R}_{\mathrm{i}} \mathrm{s}$ are completely independent of each other and that the $\mathrm{m}_{\mathrm{k}} \mathrm{s}$ are completely independent of each other. If these assumptions do not hold, then these partial derivatives are not sufficient for capturing the full sensitivity of $R_{i}$ to $m_{k}$. In reality, these assumptions will actually very rarely hold. If any correlations in the roof or the greenhouse (compartments ' $G$ ' and ' $\mathrm{H}$ ' in Fig. 1) of the HOQ exist, then it has been established that these assumptions do not apply.

In order to add formalization to the mathematical framework linking QFD and M\&S, it is first necessary to formalize the overall objective. Ultimately, the goal of engineering analyses in design and technology tradeoff is to evaluate and compare potential solutions against a set of requirements, which makes this a multi-attribute decision-making problem. In all forms of decision-making, the value of any solution against all requirements is evaluated against an overall weighted function, which will be referred to here as the total Quality Function, Q. QFD uses a similar approach, evaluating the importance of each metric based on how much it impacts a set of weighted requirements. Stated mathematically, the QFD uses a linear Q, shown in Equation (3).

$$
Q=\sum_{i=1}^{n} w_{i} R_{i}
$$

If the values in the QFD are envisioned as estimations of linear slopes, they can be thought of as partial derivatives. Thinking of them this way provides a new perspective on the results of the QFD analysis. The relative importance scores obtained then become an approximation of the partial derivatives of the total quality function with respect to each metric, and weighted according to the customer weightings, as shown in Equation (4). However, since the relationships between the metrics are not accounted for, as discussed above, this only results in the partial derivative of $\mathrm{Q}$ with respect to each $\mathrm{m}$. One advantage of using this approach is that this is not sensitive to the shape of the Q equation. While standard QFD assumes that all metrics are linearly related to all requirements, the ROSE approach to QFD allows these relationships to be any function that is differentiable. Thus, if the relationship has a quadratic slope, for example, the impact of that can be captured in the overall metric ranking, allowing decision makers to understand the impact of the values of the metrics on the ranking.

$$
\frac{\delta Q}{\delta m_{l}}=\sum_{i=1}^{n} w_{i} \frac{\delta R_{i}}{\delta m_{l}}
$$

In the case of modeling and simulation, the Multivariate Analysis of Variance may allow for the relative sensitivities of Q with respect to each metric to be calculated. However, the MANOVA does not necessarily (unless all regressors are independent) provide the sensitivity of Q to each metric. Instead, it provides the criticality of each metric to correcting capturing Q in a model of the order of the highest regressor [12]. In this way it may be useful for eliminating redundant metrics. However, because RSEs or other forms of surrogates have been obtained that have potentially higher-order relationships, it is desirable to have a way of determining these derivatives that takes into account the effect of these higher-order relations. An approximation 
of these derivatives can be found numerically using the RSEs, or even the M\&S environment itself if it is not too computationally intensive. However, ROSETTA offers an alternative way to obtain this information by thinking of the problem based on its relations between sets. In fact, if the relations between metrics are know, and the RSEs are known, then the total derivative of $\mathrm{Q}$ with respect to each metric can be found by applying Equation (5). It is interesting to note that the addition of metric sensitivities allows for the total derivative of $\mathrm{Q}$ with respect to each metric to be calculated. Since this information is actually contained in the roof of the QFD when performing qualitative analyses, replacing the QFD math with this construct will allow for the metric relations to be taken into account, even in qualitative analysis.

$$
\frac{d Q}{d m_{l}}=\sum_{i=1}^{n} \sum_{k=1}^{p} w_{i} \frac{\delta R_{i}}{\delta m_{k}} \frac{\delta m_{k}}{\delta m_{l}}
$$

Additionally, it is not necessary that the total quality function have constant weightings on the requirements as is suggested by the equation above. It is in fact possible that any differentiable quality function can be used, where the weightings obtained by differentiating $\mathrm{Q}$ with respect to each requirement. This results in Equation (6), which is the final equation used by ROSETTA to calculate the results typically found in compartment 'I' of the HOQ.

$$
\frac{d Q}{d m_{l}}=\sum_{i=1}^{n} \sum_{k=1}^{p} \frac{\delta Q}{\delta R_{i}} \frac{\delta R_{i}}{\delta m_{k}} \frac{\delta m_{k}}{\delta m_{l}}
$$

The application of this equation gives a generic way to determine the sensitivities of metrics to the full set of requirements, regardless of what information is available. This equation is insensitive as to the source of the data, so quantitative estimates can be used where M\&S surrogates, historical regressions, or physics-based equations are available, and linear qualitative estimates can be used to fill in the gaps. Thus, decision makers are not restricted in information when models are not available, but instead, can begin with a qualitative approach and update the information over time as modeling and simulation results become available to decrease uncertainty. Furthermore, users are not restricted to linear, SME-based estimates for conducting early systems engineering exercises when higher fidelity information is available for use. Using an analogous relational structure to the HOQ, the data framework for this approach is shown in Fig. 9, and can be used as a generic framework in which to track and store all data. The impact of the ROSETTA framework on a practical aerospace problem will be described in the next section.

\section{Claim 4}

In order to support the fourth and final claim, a case study will be used to demonstrate the value of ROSETTA in an aircraft design problem, by applying ROSETTA to a simplified technology tradeoff analysis for a commercial jet. In this example, two candidate technologies will be considered for inclusion on the next generation of the airliner, which will be modeled as a generic 70 passenger jet. ROSETTA has a natural application in technology tradeoff analyses. Using the quantitative relations derived above from the RSEs, it can be very quickly tested whether a set of proposed technologies will meet the goals set forth for the 
requirements. For the purpose of simplicity, the requirements will be mapped directly to the independent variables which are being impacted by the theoretical technologies, allowing a direct comparison of a QFD to M\&S.

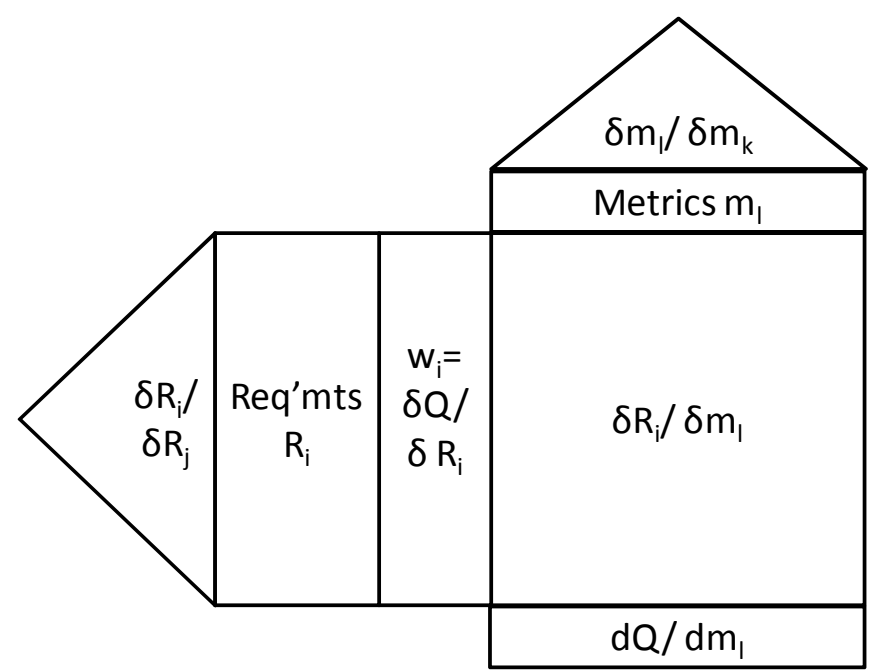

Fig. 9: Generic Relational Structure for ROSETTA. Note that the final results shown in the bottom are calculated from EQUATION, giving the total sensitivity of the total quality function $Q$ to the metrics. This process could be repeated in a QFD-style deployment to the independent variables, resulting in the total sensitivity of $Q$ to the independent variables.

For this example, a tradeoff between two candidate technologies will be considered. The first technology, a propulsive technology, will work to increase the thrust to weight ratio of the aircraft. The second technology, an aerodynamic technology, will artificially increase the effective wing area of the aircraft, thereby affecting the aspect ratio. In this fictional scenario, the technologies will be being considered with the goal of the airline being able to operate from a wider variety of airfields with smaller runways. Thus, these four requirements being considered are take-off field length, landing field length, average yield per revenue passenger mile, and direct operating cost plus interest. For the purpose of this example, it will be assumed that the requirements are evenly weighted. The impact of these technologies against these four requirements will be explored using ROSETTA, and the results of ROSETTA will be compared with the results obtained using standard QFD and M\&S.

In this example, the objective function will be to minimize $Q$, where $Q$ is given by Equation (7).

$$
Q=0.25 T O F L+0.25 \frac{T}{W}-0.25 \frac{A Y}{R P M}+0.25 A R
$$

First, a traditional QFD approach was executed. For this, the three variables that are affected by the technologies were mapped to the requirements. Each variable was then rated on a one-three-nine scale describing the level of influence each variable has against each requirement. As is common practice in standard QFD, all of the values in the body are positive, and do not reflect the direction of the impact, only the strength. The body of this QFD and the initial results of the QFD are shown in Fig. 10 . 
Although it is common practice to populate a roof in a traditional QFD, the roof is not used in the calculations and thus does not affect the results. However, the roof can be used to identify potential conflicts that might require a further trade study. In this example, one might identify a negative correlation between aspect ratio and wing area, as shown in Fig. 11, since it is known that aspect ratio varies with the inverse of wing area. While this initially makes sense, further examination of this relationship using a simple model reveals that this is an oversimplified view of the problem. Aspect ratio is defined as the wingspan squared divided by the wing area. However, the value of the wing area is not independent of the wingspan, meaning that the interaction between wind area and aspect ratio is more complicated than that captured in a simple QFD.

This more complicated relationship is illustrated using the carpet plot shown in Fig. 12: a plot of aspect ratio versus wing area for a simple rectangular wing. As can be seen from the Figure, if the area is increased by holding the span constant and changing the chord, increasing wing area does decrease aspect ratio, and the negative correlation is indeed seen. However, if the area is instead increased by holding the chord constant and changing the span, increasing area actually results in an increased aspect ratio and a positive correlation is observed. Varying both the span and the chord does not show a significant correlation between wing area and aspect ratio. This is not to imply that wing area and aspect ratio are independent, which is clearly not the case, but that the relationship between them is more complicated than can be captured in a traditional QFD. Since an equation is known that relates these two variables, it may make more sense to actually document this relationship using the equation, rather than as a simplified correlation.

The use of ROSETTA would enable the use of the actual known equation to be used in the roof of the QFD, and allows this equation to be included in the calculation of the sensitivity of the variation of these parameters on meeting the requirements. Table 1 shows the new roof of the QFD if this approach is applied.

\begin{tabular}{|c|c|c|c|c|}
\hline & wt. & $S$ & $T / W$ & AR \\
\hline TOFL & 0.25 & 3 & 9 & 2 \\
\hline LngFL & 0.25 & 3 & 1 & 0 \\
\hline Avg Yield/RPM & 0.25 & 0 & 9 & 0 \\
\hline $\mathrm{DOC}+\mathrm{I}$ & 0.25 & 0 & 9 & 0 \\
\hline Total & & 1.5 & 4.75 & 0.5 \\
\hline
\end{tabular}

Fig. 10: Traditional QFD Approach Body Results

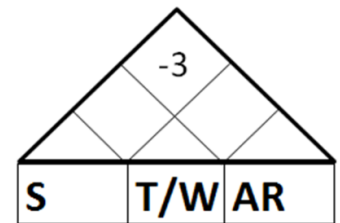

Fig. 11: Traditional QFD Roof 


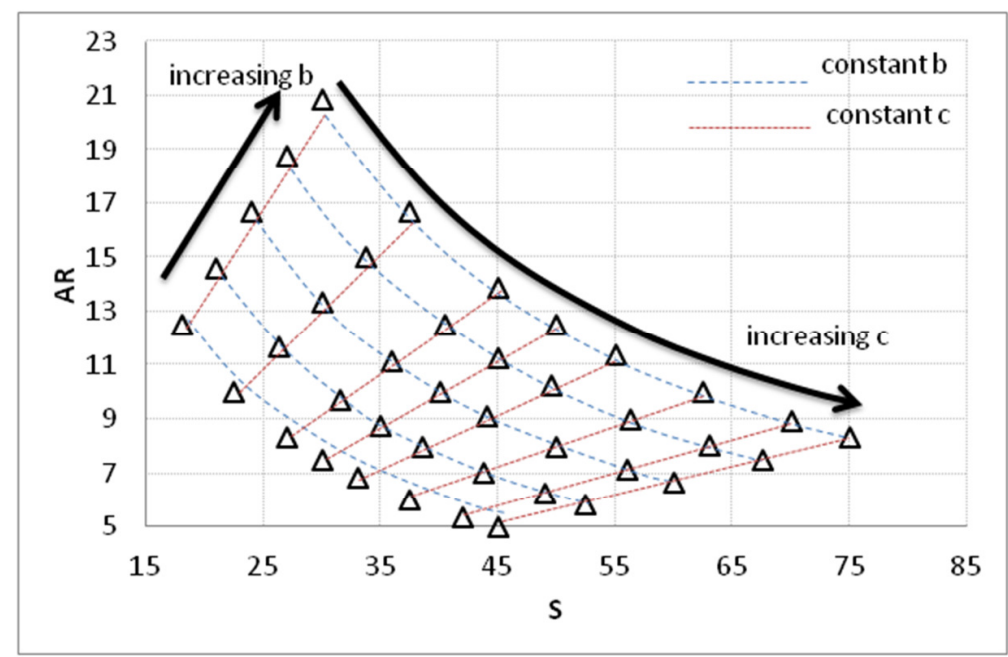

Fig. 12: Carpet Plot of S vs. AR

Table 1 Partial Derivatives of metrics with respect to metrics (Slopes obtained from definition of AR: AR=b2/S)

\begin{tabular}{|c|c|c|c|}
\hline & $S$ & TM & AR \\
\hline$\$$ & 1 & 0 & $b^{2 *} \ln ($ AR $)$ \\
\hline T/N & 0 & 1 & 0 \\
\hline AR & $b^{2 *} \ln (S)$ & 0 & 1 \\
\hline
\end{tabular}

The next step in comparing qualitative and quantitative practices is to create a quantitative model that can provide a quantitative physics-based (or equivalent in the case of cost models) approximation of the information contained in the body and the greenhouse of the QFD. For this project, a previously developed model of a generic 70 pax airliner was used. The model used in this work is a previous version of the model of the CRJ-900 documented in [13] and the modeling approach taken is similar to the approach used in that reference.

First, a design of experiments was run using this model. The design of experiments used a combination of a 287-case central composite design with a 200-case space filling design. The 287 cases from the central composite design were used to generate second order RSEs for the relevant outputs, and the 200 cases from the space filling design were used to validate the RSEs. In addition to the thrust to weight, wing area, and aspect ratio, a number of other input variables were varied as well to determine if any of these had a significant impact on the requirements of interest. The ranges used in the DoE are shown in Error! Reference source not found.. The RSEs generated were all validated to be accurate to the original model.

Once the RSEs were generated, a 10,000 case Monte Carlo Simulation (MCS) was run, in which the variables were varied across the ranges using a uniform distribution on each variable. The RSEs themselves were used to create a prediction profiler, showing the sensitivity of each response to each of the three independent variables of interest. The MCS results were used to perform a multivariate analysis, and to determine the correlation coefficients between the requirements. The prediction profiler 
serves as the quantitative equivalent of the body of the QFD and the multivariate plot represents the greenhouse. These results are shown in Fig. 13.

For each individual requirement, a screening test can also be used to help understand the impact of each variable on each requirement. The screening test assumes a linear relationship between the variables and the responses. The results of the screening test are shown in Fig. 14 in the form of tornado plots. For the purpose of this case study, only the first order terms have been included. The screening test shows similar information to prediction profiler. However, in this case, rather than show the slopes given fixed values of each of the input variables, the screening test shows the impact and direction of each input variable to the total variability of the requirement. Thus, unlike the prediction profiler, these results are not dependant on the fixed values of other metrics, but rather summarize the sensitivity across the entire range for which the metrics were varied. In this case, all of the variables included in the DoE are shown in the results. This gives a way to directly compare the results across each row of the prediction profiler. The three variables of interest are highlighted in each figure. At first glance, one might draw the conclusion that the thrust to weight has the most impact on the overall requirements space. However, closer examination suggests that while it has a strong desirable influence on take-off field length, it has an undesirable influence on the average yield per revenue passenger mile and the direct operating cost plus interest. Therefore, while a small change in thrust to weight will result in significant changes across the requirements space, not all of these significant changes will be desirable, and thus this may not be the best technology to choose.

From the RSEs, the average slope of the relationship between each of the independent variables and the requirements can be calculated. This can be used as an estimate of the sensitivity in the ROSETTA relational structure. These estimated average slopes are shown in Table 3. There are several important observations to be made from these results. First, the sensitivities obtained through modeling and simulation has both a direction and a magnitude. Thus, it is possible to determine not only if each variable will impact the requirement, but whether that impact will be positive or negative. Furthermore, it can be noted that the relative magnitudes of the slopes have a much greater variation than would be implied by the one-three-nine scale often used in QFD. For example, a small change in thrust to weight will have a much larger impact on these requirements than a small change in the wing area. This suggests that it may be desirable to use estimated sensitivities in qualitative analyses rather than a simple scale. These sensitivities could be estimated from similar, previous studies or from historical data. 
Table 2 DoE Ranges for 70 pax Model

\begin{tabular}{|l|r|r||}
\hline & Low & High \\
\hline Wing Area & 750 & 830 \\
\hline TWR & 0.33 & 0.38 \\
\hline AR Wing & 7.35 & 9 \\
\hline Taper Wing & 0.3 & 0.4 \\
\hline t/c root & 0.11 & 0.13 \\
\hline t/c tip & 0.11 & 0.13 \\
\hline Sweep & 26 & 35 \\
\hline ARHT & 3 & 5 \\
\hline Taper HT & 0.4 & 0.55 \\
\hline HT t/c & 0.11 & 0.13 \\
\hline HT area & 170 & 250 \\
\hline AR $\vee T$ & 0.8 & 1.4 \\
\hline Taper $V T$ & 0.5 & 0.7 \\
\hline VT t/c & 0.11 & 0.13 \\
\hline VT area & 100 & 180 \\
\hline
\end{tabular}

Another important note is that each of these estimated slopes is calculated based on the assumption of a fixed value for the other metrics. In the case of Table 3, the average value has been assumed for each metric in the approximation of the average slope. However, if a different value is assumed, it is probable that these slopes would change because of the coupling between metrics. Since it was observed previously that wing area and aspect ratio are not unrelated, it is expected that variation on the assumption of one of these would affect the relative sensitivities of the other. A way to account for this coupling is required and is currently being explored as part of this initiative.

Finally, the greenhouse of the QFD is quantitatively approximated by examining the results of the MCS. The correlations between the requirements can be approximated from the MCS data set, and are shown in Table 4. It can be noted that the average yield per revenue passenger mile and the direct operating cost plus interest are highly correlated. Thus, it may only be necessary to track or model one of these requirements. Being able to make this kind of observation may help to reduce future modeling needs and costs. There is a less significant but still notable positive correlation between takeoff and landing field length.

However, correlation alone does not give the complete picture. The correlation only represents the linearity of the relationships. It is still important to understand the slope, or sensitivities. For example, image that one decided to only model direct operating cost plus interest to save modeling effort. If sensitivity between this and the average yield per revenue passenger mile were both know, it would then be possible to actually approximate a value for the average yield per revenue passenger mile using this relationship. Furthermore, using the correlation information, it would be possible to determine the confidence in this estimate. 


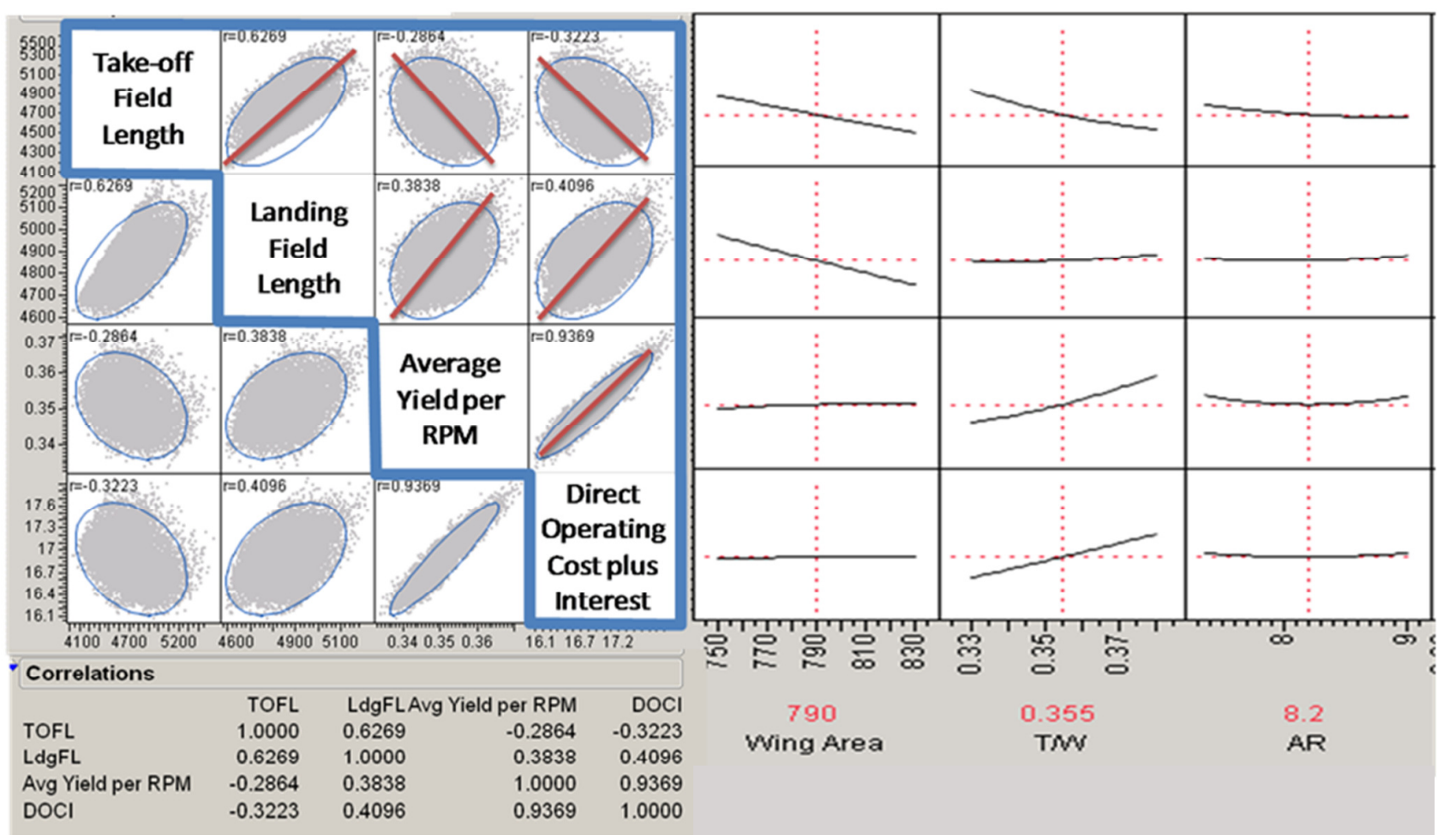

Fig. 13: Modeling and simulation results, including multivariate plot showing requirements correlations and prediction profiler showing the sensitivity of requirements to the metrics

By combining the results shown above in the ROSETTA framework using Equation (6), the total derivates of Q with respect to each metric can be approximated. In this case, these derivates will not be a single number but rather an equation that is dependent on the geometry of the aircraft and which are robust to changes in the aircraft design, meaning that they still apply as the design is updated, provided that the design does not exceed the ranges used to create the RSEs. Thus, it will be possible to see the impact of design changes or uncertainty in the performance of new technologies on the total quality function, and thus on meeting the overall requirements. The results obtained using ROSETTA are given in Equations (8) through (10), and shown graphically in Fig. 15. These results are significantly different from those obtained using the traditional QFD approach described above. First, the direction of the impact of each metric on $\mathrm{Q}$ is shown. In this case, all three metrics considered show a negative impact, meaning that they will work to reduce the magnitude of Q, which is a desirable effect. However, if one of these had the opposite effect, ROSETTA would allow the user to see this, thereby providing important information regarding not only how sensitive the requirements are to that metric, but also whether an increase in that metric has a net positive or negative effect on the requirements space. The uncertainty bars represent the range of possible values for the derivatives of $\mathrm{Q}$ with respect to each metric assuming that each metric is allowed to vary according to the ranges included in the DoE.

$$
\frac{d Q}{d S}=-12.5 * b^{2} * \ln (A R)
$$




$$
\begin{gathered}
\frac{d Q}{d\left(\frac{T}{W}\right)}=-1081 \\
\frac{d Q}{d A R}=-100 * b^{2} * \ln (S)
\end{gathered}
$$

A second observation is that the ranking of the metrics is opposite of what was predicted using QFD. In this case, the results show that in the ranges considered, the aerodynamic technology will have a significantly larger impact on the metrics of interest as compared to the propulsive technology. In fact, the ranking is fully reversed from what was predicted by the QFD. A direct comparison of the rankings obtained with QFD and ROSETTA is shown in Fig. 16. For this comparison, the absolute value of the ROSETTA results was used, and both the QFD and ROSETTA results were normalized on a zero to one scale. It can be concluded that traditional QFD was not an appropriate choice for a first order study in this area. Without taking into account both the direction and the magnitude of each variable on each requirement, the results obtained were misleading. While QFD did successfully identify that thrust to weight had the most significant impact on the requirements, it did not include enough information to determine if it the impact was desirable or undesirable.

Table 3 Partial Derivatives of requirements with respect to metrics (Slopes obtained from M\&S results, linear in range of interest)

\begin{tabular}{|c|c|c|c|}
\hline & S & T/W & AR \\
\hline TOFL & -7.5 & -1200 & -100 \\
\hline LndgFL & -5 & 1000 & 0 \\
\hline Avg Yield/RPM & 0 & 0.6 & 0 \\
\hline DOCI & 0 & 18 & 0 \\
\hline
\end{tabular}

Table 4 Correlations between requirements, obtained from modeling and simulation results

\begin{tabular}{|l|r|r|r|r|}
\hline & TOFL & LndgFL & Avg Yield/RPM & DOCI \\
\hline TOFL & 1 & 0.6269 & -0.2864 & -0.3223 \\
\hline LndgFL & 0.6269 & 1 & 0.3838 & 0.4096 \\
\hline Avg Yield/RPM & -0.2864 & 0.3838 & 1 & 0.9369 \\
\hline DOCI & -0.3223 & 0.4096 & 0.9369 & 1 \\
\hline
\end{tabular}


Take-off Field Length

Landing Field Length

\begin{tabular}{|c|c|c|c|}
\hline Term & Contrast & Term & Contrast \\
\hline TMN & -252.189 & Wing Area & -141.521 \\
\hline Wing Area & -231.634 & Sweep & .69 .414 \\
\hline AR & -100.023 & Wing Area HT & 29.048 \\
\hline Sweep & -86.741 & TOC3 & 27.207 \\
\hline Wing Área HT & 35.624 & TOC1 & 29.183 \\
\hline TOC1 & 32.704 & Wing Area VT & 24.249 \\
\hline TOC3 & 35.458 & $T N$ & 18.181 \\
\hline Wing Area VT & 31.016 & TR & 9.415 \\
\hline TR & 11.707 & TRHT & 5.978 \\
\hline TRHT & 7.466 & TRVT & 4.859 \\
\hline TRVT & 6.351 & AR & 3.563 \\
\hline TOCHT & 2.835 & TOCHT & 2.339 \\
\hline ARHT & 2.034 & ARVT & 1.320 \\
\hline ARVT & 1.691 & TOCVT & 0.933 \\
\hline TOCVT & 1.507 & ARHT & 0.601 \\
\hline \multicolumn{2}{|c|}{ Average Yield per Revenue Passenger Mile } & \multicolumn{2}{|c|}{ Direct Operating Cost plus Interest } \\
\hline Term & Contrast & Term & Contrast \\
\hline$T M$ & 0.006997 & $T M$ & 0.398255 \\
\hline Sweep & -0.003854 & Sweep & -0.213281 \\
\hline Wing Area HT & 0.001582 & Wing Area HT & 0.087813 \\
\hline Wing Area VT & 0.001444 & TOC3 & 0.084059 \\
\hline TOC3 & 0.001435 & TOC1 & 0.090532 \\
\hline TOC1 & 0.001542 & Wing Area VT & 0.075157 \\
\hline Wing Area & 0.000695 & TR & 0.028139 \\
\hline TRHT & 0.000529 & Wing Area & 0.024445 \\
\hline TR & 0.000434 & TRHT & 0.018404 \\
\hline TRVT & 0.000340 & TRVT & 0.015985 \\
\hline ARHT & 0.000333 & AR & -0.013654 \\
\hline AR & -0.000316 & TOCHT & 0.006310 \\
\hline TOCVT & 0.000111 & ARHT & 0.004608 \\
\hline ARVT & 0.000076 & ARVT & 0.003827 \\
\hline TOCHT & -0.000037 & TOCVT & 0.003616 \\
\hline
\end{tabular}

Fig. 14: Screening Test Results 
Further analysis using the ROSETTA framework showed that increasing the thrust to weight actually had an overall undesirable effect on the requirements space, thus suggesting that the propulsion technology was not an appropriate choice. Even if the modeling and simulation had not been available and subject matter experts were instead used, including the direction of the impact would have reduced the probability of drawing an incorrect conclusion from the initial QFD.

By comparing the results of ROSETTA and the traditional QFD to the results of the M\&S, it can been seen that the results of ROSETTA more closely match what is predicted by modeling and simulation. While thrust to weight does have a significant impact on each of the requirements, increasing thrust to weight improves performance on some requirements while degrading performance on others. Thus, its total impact on improving the requirements space overall is low. Increasing the effective wing area, however, has a strong impact on the field length requirements, and a smaller impact on the economic requirements. In all cases, however, it has either a desirable impact or no impact on the requirements. Thus, overall, it is a much bigger driver for improving your requirements space. Because traditional QFD does not account for the direction of the sensitivities, it predicted thrust to weight to have an overall greater impact, and would have, in this case, led designers to choose a technology that would not have achieved the desired goals. The ROSETTA framework allows designers to take into account the direction and the relative magnitudes of these sensitivities, and thus can provide a result that more closely simulates the results that would be obtained from the M\&S.

The observations made by comparing the qualitative and quantitative approaches to this simple aircraft example highlight the need for a more rigorous and formalized approach to making early estimations in the design process. If qualitative methods could be adapted to be able to include equations and historical data where they are known and use subject matter expert information where they are not, it would be possible to increase the fidelity and confidence of these early-phase systems engineering results. The ROSETTA framework offers an alternative approach to the QFD that is more appropriate for system design and which is more flexible, allowing users to include as much available information as possible to increase confidence in results. Furthermore, the results obtained using the ROSETTA framework are more robust to requirements creep and to design uncertainty, and allow a more complete picture to be developed in the early phases of design. 


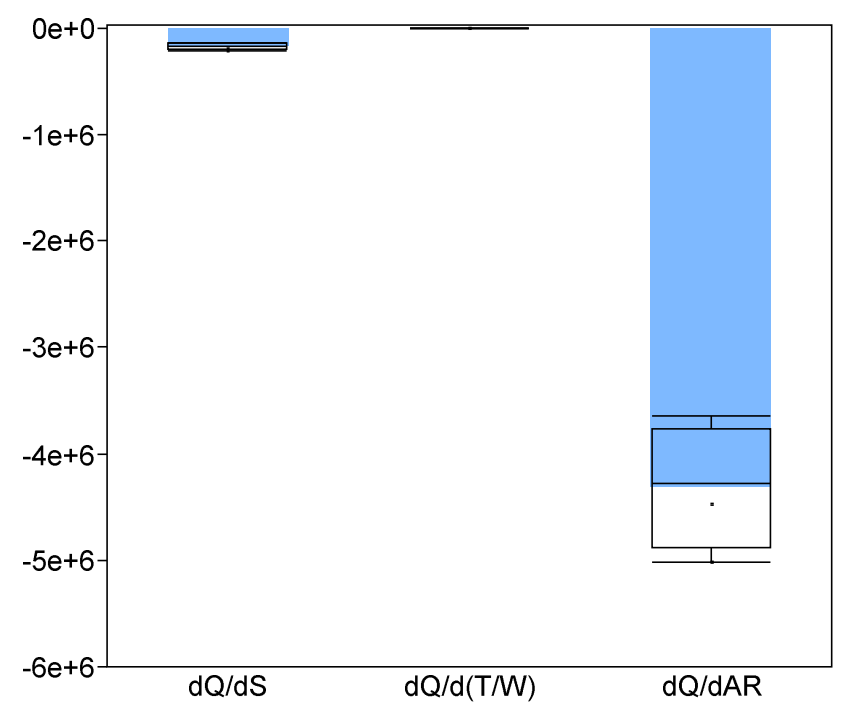

Fig. 15: ROSETTA results. Note that these prioritizations are reversed from what was expected using the traditional QFD approach. Furthermore, it shows that while both technologies will act to minimize Q, the aerodynamic technology will have a significantly larger impact, contrary to the predictions of the QFD. The uncertainty in the results based on the range of values for each metric is shown using the error boxes, which represent the full range of possible outcomes.

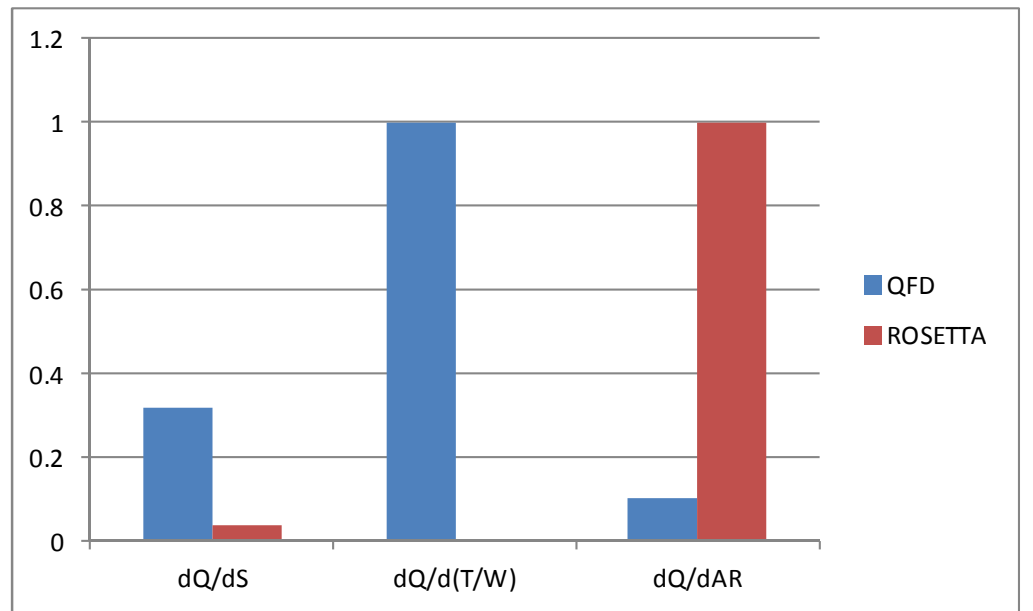

Fig. 16: Comparison of ROSETTA and QFD results. The absolute value of the ROSETTA results is used for this comparison. Both sets of results have been normalized to a zero to one scale and using the maximum value obtained in that set of results.

\section{Concluding Remarks}

ROSETTA provides a framework to strengthen and make more formal the relationship between QFD, modeling and simulation, and theoretical mathematics, and allow translation between these three approaches to engineering problems. While these types of approaches are typically implemented at different phases in the systems engineering process, clearly understanding how they are related can provide insight as to how to improve the overall systems 
engineering process. ROSETTA brings together approaches that are typically executed independently to strengthen each method and improve the overall design process. This paper focused on several identified deficiencies in QFD for performing technology tradeoff analyses, primarily the assumption of linear relationships, the exclusion of the roof from calculations, and using correlations without considering sensitivities in the roof and the greenhouse.

The proposed ROSETTA framework addresses these deficiencies by providing a structured and generic mathematical framework that can capture the relationships contained in the QFD in a more complete manner. ROSETTA exploits transformations to create a modeling and simulation-inspired approach to the collection and integration of qualitative and quantitative information in early systems engineering. ROSETTA leverages key aspects of the ROSE methodology to enable this integration of qualitative and quantitative information and create a more formal representation of QFD that can be used to support early-phase engineering decision making.

The data structure proposed by ROSETTA provides a generic framework for engineering analysis that allows qualitative and quantitative data to be mixed in the decision space, allowing decision-makers to continually update their information as data becomes available and revisit previous decisions to ensure that the assumptions that led to that decision are holding. If they do not hold, the framework is also able to be used to determine if the decision is still a good one, and if not, determine the appropriate course of action. It allows the impact of uncertainty to be captured throughout the design process, and the uncertainty to be reduced continually. The ROSETTA framework has the potential to be robust to any type of application as there are no assumptions made about the types of relationships that exist in the problem space.

The case study examined in this paper demonstrates the significant impact of ROSETTA on early systems engineering results and the potential of the approach. The application of ROSETTA showed a significant deviation in results as compared to traditional QFD, and these results were shown to be more in line with what was expected based on the results of the M\&S. This case study only begins to explore the full potential of the ROSETTA framework, and future applications can be used to continue to provide innovations in this new approach. The application of ROSETTA to this commercial jet trade study offers a new approach to the systems engineering community to more rigorously tackle a wide range of system and system of systems engineering problems.

\section{References}

[1] ReVelle, Jack B., Moran, John W. and Cox, Charles A. The QFD Handbook. New York, NY : John Wiley \& Sons, Inc, 1998. 
[2] Shinbara, Tim J. "The Application of High Value SIBR Programs to F-35 Lightning II NGC SOW," prestented Mar. 31, 2011

[3] Meyers, Raymond H., Montgomery, Douglas C. and Anderson-Cook, Christine M. Response Surface Methodology: Process and Product Optimization Using Designed Experiments Hoboken, NJ : John Wiley \& Sons, 2009.

[4] Dickerson, Charles E. and Mavris, Dimitri N. "Relational Oriented Systems Engineering (ROSE): Preliminary Report" in Proc. 6th Annual IEEE SoSE Conference, Albequerque, 2011

[5] Breyfogle, F. W. Implementing Six Sigma, Second Edition: Smarter Solutions Using Statistical Methods. s.l. : Productivity Press, 2003.

[6] Hauser, John R and Clausing, Don. s.l. The House of Quality. 1988, The Harvard Business Review, Vols. May-June, pp. 63-73.

[7] Olhanger, Jan and West, B. Martin. The House of Flexibility: Using the QFD Approach to Deploy Manufacturing Flexibility. 1, 2002, International Journal of Operations and Production Management, Vol. 22, pp. 50-79.

[8] T Mazur, Glen H. Yokohama The Application of Quality Function Deployment (QFD) to Design a Course in Total Quality Management (TQM) at the University of Michigan College of Engineering. Proceedings of International Conference on Quality-1996, 1996.

[9] Mavris, Dimitri N and Kirby, Michelle. Technology Identification, Evaluation, and Selection (TIES) for commercial transport aircraft. San Jose, CA : s.n., 1999. 58th Annual SAWE Conference.

[10] Bray, James H. and Maxwell, Scott E. Multivariate Analysis of Variance. Newbury Park, CA : Sage Publications, 1985. Quantitative Applications in the Social Sciences, Issue 54.

[11] Dickerson, Charles E. and Mavris, Dimitri N. Archtiectures and Principles of Systems Engineering. 2010 : Taylor and Francis Group, 2010.

[12] Anderson, T. W. An Introduction to Multivariate Statistical Analyses John Wiley and Sons, 2003

[13] Jimenez, Hernando; Pfaender, Holger; Mavris, Dimitri. "System-wide Fleet Assessment of NASA Environmentally Responsible Aviation (ERA) Technologies and Concepts for Fuel Burn and CO2," in Proc. 11th AIAA Aviation Technology, Integration, and Operations (ATIO) Conference, including the AI, Virgina Beach, 2011, AIAA 2011-6882 